\title{
Single enhancing computerized tomography-detected lesion in immunocompetent patients
}

\author{
RaVindra Kumar Garg, M.D., D.M. \\ Department Of Neurology, King George's Medical College, Lucknow, India
}

\begin{abstract}
Single enhancing computerized tomography (CT)-documented lesions may occur in several infectious and neoplastic diseases of the central nervous system and are the most common radiological abnormality seen in patients with acute-onset seizures in India and many other developing countries. Similar CT-documented lesions have also been reported in the developed world where these lesions are often considered to be caused by neoplasms or tuberculoma. Histopathological studies in India and even in some developed countries revealed that neurocysticercosis (NCC) is the most likely cause of these lesions provided they fulfill a rigid set of clinical and radiological criteria. Single cysticercus granuloma measures less than $20 \mathrm{~mm}$ in diameter, may be associated with cerebral edema not severe enough to produce midline shift, and occur in patients with seizures, normal neurological status, and no evidence of active systemic disease. When these lesions resolve spontaneously, either disappearing or changing into a calcified nodule, the diagnosis of NCC is very likely. The second most common cause of these CT-detected lesions is tuberculoma; in patients with these lesions similar clinical and neuroimaging features are also present. Few authors believe that in poor and developing countries, where both tuberculosis and NCC are common, it is difficult to differentiate between tuberculoma and a single cysticercal granuloma. The most interesting feature of these solitary enhancing lesions is their spontaneous disappearance within weeks or months. Some lesions "heal" by becoming calcified. These patients require only antiepileptic therapy, and this medication may be withdrawn safely after the lesion has resolved on CT scanning. In several studies provision of anticysticercal drugs has been attempted, but because of conflicting results, their role in the management of these single lesions is uncertain. Neurosurgeons have a very limited role to play. In very rare situations, if the lesion increases in size and if focal neurological deficits manifest or seizures are uncontrolled, a brain biopsy sample may be required and histopathological evaluation performed to establish the correct diagnosis.
\end{abstract}

\section{KEY WoRDS • cysticercosis • computerized tomography • epilepsy • neurocysticercosis • tuberculoma • seizure}

Single enhancing lesions visualized on CT scanning are the most common radiological abnormality in Indian patients with new-onset seizures ${ }^{17}$ (Fig. 1). In 1980, Tandon and Bhargava ${ }^{48}$ first reported these lesions; at that time these CT-enhancing lesions were presumed to be tuberculoma and often were treated with empirical antituberculous drugs. Subsequently, histopathological studies of brain tissue biopsy samples have suggested that, in majority, single CT-enhancing lesions represent dying cysticercal lesions (larval stage of tape-worm Taenia solium). ${ }^{39}$ These single CT-enhancing lesions often resolve spontaneously. ${ }^{17}$ Considerable controversy persists about their cause and appropriate management. In this article current understanding about these lesions is reviewed and an approach toward management discussed.

Abbreviations used in this paper: AVM = arteriovenous malformation; $\mathrm{CNS}=$ central nervous system; $\mathrm{CT}=$ computerized tomography; $\mathrm{ICP}=$ intracranial pressure; $\mathrm{MR}=$ magnetic resonance; $\mathrm{NCC}=$ neurocysticercosis

\section{EPIDEMIOLOGY OF THE SINGLE CT-ENHANCING LESION}

The precise incidence and prevalence of single enhancing CT-documented lesions, in India and other parts of the world, are not known. All data available in India are from hospital-based studies. Wadia, et al., ${ }^{50}$ studied 150 patients with simple partial seizures, and CT scanning revealed single enhancing lesions in approximately $26 \%$. The incidence of these lesions was higher among children; $40 \%$ of patients were younger than 15 years of age. Misra, et al. ${ }^{24}$ from North India, studied 1023 patients with partial seizures and obtained plain and contrast-enhanced CT scans in all. A single enhancing CT-demonstrated lesion was found in approximately $50 \%$ of patients (513 cases) ${ }^{24}$ In a study from South India Murthy et al. ${ }^{26}$ reported these CT lesions in $23.4 \%$ of 2531 patients with all types of epilepsies. Garg and $\mathrm{Nag}^{16}$ observed a higher incidence (72\%) of single enhancing lesions in children and adolescents when CT scanning was performed after the first seizure.

Although initially it was thought that these lesions are 
found only in individuals from the Indian subcontinent, similar lesions were later reported from other parts of the world. Single enhancing lesions are frequently seen in Latin American countries and are invariably treated as cysticercal granuloma. ${ }^{8}$ These lesions have also been reported in studies conducted in several developed countries such as Australia, the United States, and the United Kingdom. ${ }^{20,23,47}$ Wadley, et al., ${ }^{51}$ recently reported a series of six patients from United Kingdom with single enhancing lesions. In all six patients the referral diagnoses were either tumor or tuberculoma. In the majority, the lesions had spontaneously disappeared. These authors concluded that patients harboring single enhancing CT-documented lesions, even in nonendemic regions, need careful observation because their lesions may disappear spontaneously and patients may be saved from unnecessary neurosurgery.

\section{RADIOLOGICAL FEATURES}

The characteristic ring or disc-enhancing CT lesions are seen after intravenous contrast administration. Plain CT scans, at times, demonstrate some abnormality. The most common abnormality observed on plain scans is irregular low attenuation areas of vasogenic cerebral edema. Infrequently, a tiny speck of calcification is demonstrated within the area of hypodensity. Using newer-generation CT machines, it is possible to see the outline of complete cystic lesion with an extramural nodule. ${ }^{13}$ Plain CT scanning may not reveal any abnormality. ${ }^{6,15,17}$

After contrast administration, there is a ring or a homogeneous disclike enhancement within the region of hypodensity. The enhancing lesions observed on these CT scans are usually less than $20 \mathrm{~mm}$ in diameter; they are surrounded by a varying amount of perifocal edema. Occasionally, the edema may be severe enough to produce midline shift. An enhancing or a calcified eccentric dot (presumed to be a scolex of cysticercal larva) can be seen within the ring lesion (Fig. 1). Single CT-documented lesions can be seen throughout the cerebral hemispheres, more commonly situated superficially in the gray matter or at the junction of gray matter and white matter. Parietal lobes are the most common location for these le-

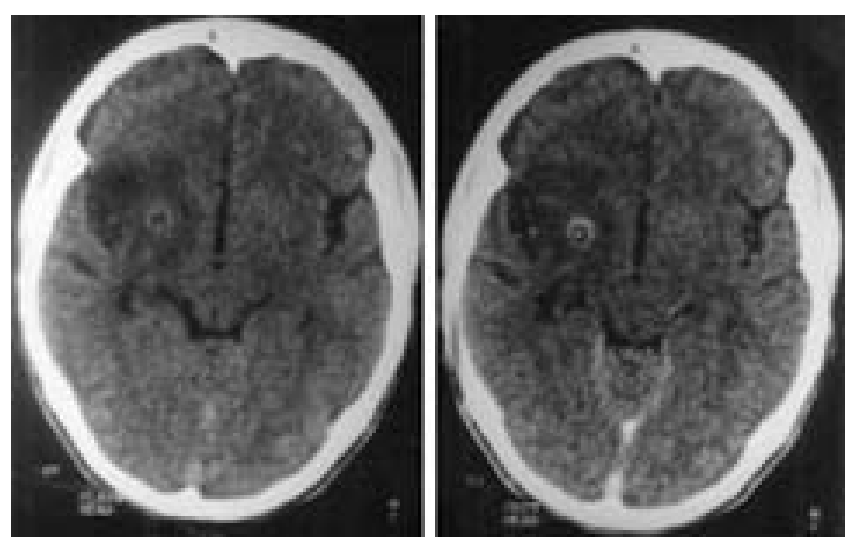

Fig. 1. Plain (left) and contrast-enhanced (right) CT scans revealing a ring-enhancing lesion. sions. Frontal and occipital lobes are the other frequent sites. ${ }^{6,15,17,31}$

\section{RADIOLOGICAL DISAPPEARANCE OF THE ENHANCING LESION}

The most remarkable feature of single enhancing lesions observed on CT scanning is their complete spontaneous disappearance in the majority of patients, as well as their occasional significant reduction in size in others. The edema surrounding the lesion is usually the first to resolve. Later, the lesion may disappear completely, leaving no residue, or it may leave a tiny speck of calcification at the former site of the lesion. In some patients the granulomatous lesion transforms into a calcified nodule. ${ }^{17,31}$ (Fig. 2)

Controversy exists regarding the exact time that these CT-enhancing lesions disappear. In several retrospective studies the authors have suggested that spontaneous resolution may vary from as early as 6 weeks to as late as 75 weeks. ${ }^{15,42,43,50}$ Even greater persistence has been observed. Various authors have noted a wide variation in the rates of complete resolution. The rate of disappearance has ranged from 22 to $100 \%$ at 12 weeks after detection on the first CT scan. Addressing this same issue, Rajshekhar, ${ }^{32}$ in a prospective study of 210 patients, observed that single enhancing lesions completely resolved at different time intervals. At 3 months only 19\% of lesion had completely resolved; at 1 year approximately $63 \%$ had disappeared. In another prospective study, which supports majority opinion, Singh, et al., ${ }^{46}$ observed that approximately $73 \%$ of similar-appearing lesions had disappeared within 2 months of their first CT documentation. Because of these conflicting observations, it is very difficult to recommend guidelines concerning need and timing of obtaining follow-up CT scans in these patients.

\section{ETIOPATHOGENESIS OF THE SINGLE ENHANCING LESION}

Since these lesions were first described, their precise origin has remained controversial. Several origins have been postulated, from time to time, to explain the cause of these single enhancing lesions (Table 1).

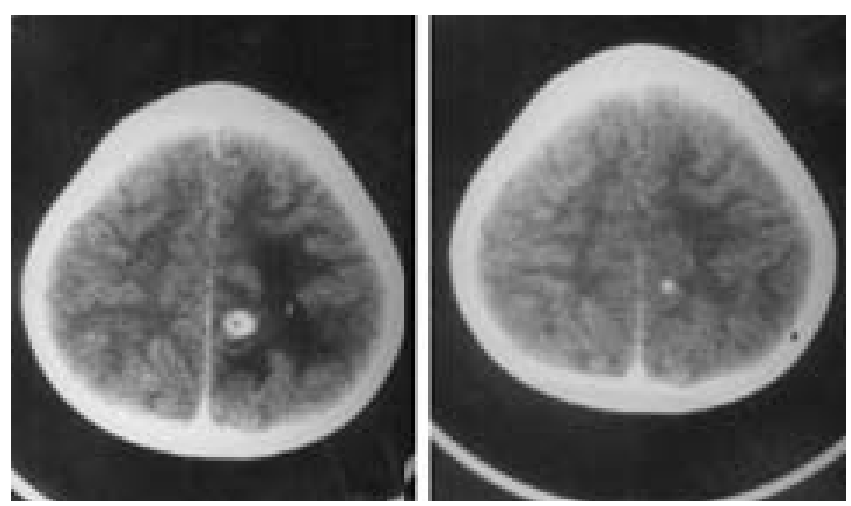

Fig. 2. Computerized tomography scans revealing a ring-enhancing lesion (left) and calcification of the lesion (right). 
TABLE 1

Summary of causes of single enhancing lesions demonstrated on CT scanning

common
NCC
tuberculoma
uncommon
glioma
secondary disease
cryptic AVM
brain abscess
larva migrans
sarcoidosis
small infarct
focal encephalitis
in immunocompromised patients
toxoplasmosis
CNS lymphoma
fungal granuloma

\section{Postictal Phenomenon}

Until recently one of the major points of controversy was whether these lesions were cause or effect of the associated seizure disorder. Several biochemical and physiological changes occur at the site of abnormal neural activity during or following partial seizure. It was thought that the changes noted in neurons, blood vessels, and the blood-brain barrier had resulted in ring- or disclike enhancement on CT scanning. ${ }^{18,19}$

\section{Intercranial Tuberculoma}

In initial reports, authors determined that single enhancing lesions were tuberculoma, based on several presumptions. Intracranial tuberculoma were common intracranial space-occupying lesions in this part of the world. Tuberculoma and tuberculous abscess constituted approximately 10 to $20 \%$ of all intracranial masses. Similar lesions documented on CT scanning were often seen in association with tuberculous meningitis (Fig. 3). A favorable response to empirical antituberculous treatment was observed. Finally, tuberculosis is a highly endemic disease in India. ${ }^{21,48}$

\section{Neurocysticercal Cysts}

A major breakthrough in the understanding of these single CT-enhancing lesions came when Chandy, et al., reported obtaining CT-guided stereotactic biopsy samples. Histopathological examination of these brain tissue samples showed cysticercal granuloma in the majority of patients. In another study of 51 patients Rajshekhar, et al., ${ }^{39}$ documented cysticercal granulomas in 25 patients and tuberculoma in six; in all cases these findings were confirmed. Of the remaining 20 patients, 12 patients harbored parasitic granuloma (cysticercal lesion not definite), six patients nonspecific inflammation, and one patient each had dystrophic calcification and secondary metastasis. The authors concluded that the majority of single enhancing CT lesions are caused by NCC; however, other diseases such as tuberculoma should always be considered in the differential diagnosis.

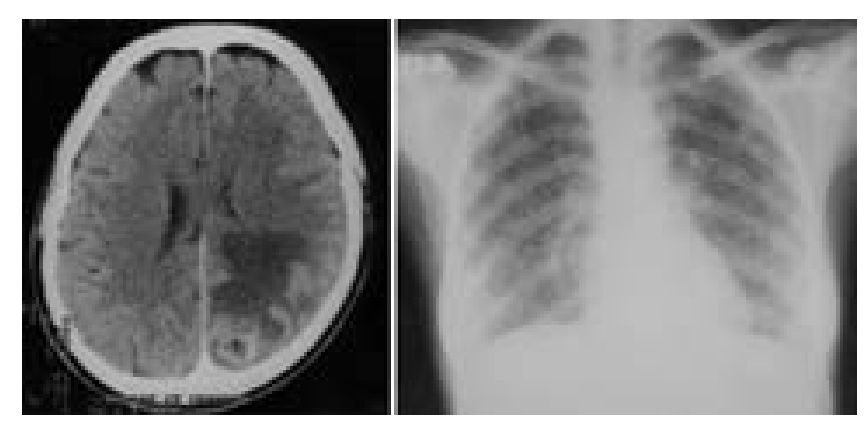

Fig. 3. Left: Contrast-enhanced CT scan demonstrating a ringenhancing lesion. Right: Radiograph of the chest obtained in the same patient, revealing miliary tuberculosis.

\section{Other Diseases Causing Single Enhancing Lesions}

Various other presumptive diagnoses such as glioma, larva-migrans infection, microabscess, secondary metastasis, small infarct, focal encephalitis, and cryptic AVM have been suggested but none is consistently demonstrated in histopathological studies. ${ }^{17,31}$

\section{PATHOLOGICAL CHARACTERISTICS}

A parenchymal cysticercal cyst of the brain passes through four stages of natural evolution. After entering in to the brain parenchyma, the parasite develops into a "vesicular stage" in which the cysts are viable and elicit very little inflammatory response in the surrounding brain tissue. On CT scanning viable cysticercal lesions appear as rounded, circumscribed, hypodense lesions, and contrast enhancement is absent. After a variable period of time the parasite loses its viability either because of aging, inability of larva to become adult, or immunological factors particular to the host, and it enters into the next stage. The second phase is the "colloidal stage" in which inflammatory changes develop in the cyst wall and surrounding brain parenchyma. Transparent cystic fluid is replaced by jellylike whitish material, which is surrounded by a fibrous capsule. This dying stage of larva is referred to as cysticercal granuloma. In this stage, CT scanning demonstrates a ring-enhancing lesion. Progressive reduction in the size of the cyst and scolex and mineralization of cystic fluid lead to development of a "granular-nodular" stage in which the larva appears as a disc-enhancing lesion on contrast-enhanced CT. In the last "calcific stage" the lesion becomes completely mineralized and appears as a hyperdense calcified nodule on plain CT scanning. At this stage there is no contrast enhancement and surrounding edema is also absent because of abatement of inflammation. ${ }^{11}$

Rajshekhar, et al., ${ }^{34}$ have attempted to demonstrate the presence of a viable parasite within the single CT-enhancing lesions. They performed histopathological examination of 43 brain tissue biopsy samples and were successful in demonstrating cystic lesions containing parts of an intact or degenerated larva in 22 patients. Inflammatory cavitary lesions, in which there was no definite evidence of parasite, were observed in 13 patients, whereas noncavitary hyalinized fibrous nodules with inflammation were 
found in the remaining eight. Of the 22 specimens in which there was definite evidence of cysticercosis, only lesions in two patients were shown to have an entire parasite within the granulomatous lesion. It was not possible to predict the presence of an intact parasite within the granuloma on the basis of clinical and radiological features.

In a more recent article, Chacko, et al., ${ }^{4}$ reported that, in a few patients, when no intact parasite or parasitic parts were observed within a granuloma, it was possible to demonstrate the presence of small ovoid masses corresponding morphologically to the intracorporeal vacuoles of a cysticercal larva. These structures were found to lie within the cavitary space of granuloma. The authors concluded that even the presence of calcareous residues of parasites may be the only evidence of cysticercal origin in some of the granulomas.

\section{CLINICAL FEATURES}

The patients in whom CT scanning reveals single enhancing lesions usually present with new-onset seizures. The seizures are often partial (motor $>$ sensory) with or without secondary generalization. If a lesion is located in the occipital lobe, seizure is often preceded by visual aura, and in frontal lobe lesions an "adversive attack" is frequently observed. Cases of complex partial seizures are rare. Few patients present with partial status epilepticus. Several episodes of seizure occur in clusters, within a span of 2 to 3 days. Infrequently, Todd paralysis, which resolves within 24 hours, is observed. ${ }^{15,35,50}$ Another major complaint in patients with single enhancing lesions is headache. A large number of patients presenting with seizure experience persistent or episodic headache during the interictal period. ${ }^{15,17}$ In some patients, severe episodic headache may be the only presenting complaint. In these patients the headache can be of such severe intensity so as to mimic that of a subarachnoid or an intracerebral hemorrhage. The fundus is usually found to be normal. ${ }^{33,35}$ Infrequently, the headache may be part of a frank increase in ICP. Patients experience headache, vomiting and papilledema. In this variety of headache the enhancing lesions have been noted in subcortical locations. Headache and other signs of raised ICP improve after spontaneous resolution of the lesion. Oral corticosteroid medication helps to relieve headache in the acute stage.${ }^{14}$ Patients in whom CT scanning demonstrates a single enhancing lesion in the brainstem invariably suffer headache and exhibit other brainstem signs. ${ }^{22}$ Focal neurological deficits in the absence of seizures are rare and, at times, may be the sole manifestation. ${ }^{14}$

\section{DIAGNOSIS OF THE SINGLE ENHANCING LESION}

\section{Cysticercal Granuloma and Tuberculoma}

The distinction between cysticercal granuloma and tuberculoma is controversial, often associated with single enhancing CT-documented lesions. This is because the clinical and imaging features are quite similar; both diseases are common in endemic areas and may coexist in the same patient.
TABLE 2

Diagnostic criteria for cysticercal granuloma*

\begin{tabular}{c}
\hline \multicolumn{1}{c}{ Criteria } \\
\hline clinical \\
seizure (partial or generalized) as initial symptom \\
no persistent raised ICP \\
no progressive neurological deficit \\
no active systemic disease \\
CT picture \\
solitary, contrast-enhancing lesion \\
20-mm diameter lesion \\
no severe cerebral edema (no midline shift)
\end{tabular}

* As established by Rajshekhar and Chandy (1997).

Rajshekhar, et al. ${ }^{39}$ have attempted to differentiate between these two entities on the basis of clinical and imaging features. Based on these findings and their experience, Rajshekhar and Chandy ${ }^{38}$ suggested that cysticerci are usually round in shape, $20 \mathrm{~mm}$ or smaller in size, with ring enhancement or a visible scolex; cerebral edema severe enough to produce midline shift or focal neurological deficit is not seen. Tuberculomas, by contrast, are usually irregularly shaped, solid, and greater than $20 \mathrm{~mm}$ in size. They are often associated with severe perifocal edema and focal neurological deficit ${ }^{38}$ (Table 2). This distinction is important because parenchymal cysticercosis is a benign and self-limiting condition, whereas tuberculoma is an active infection requiring prolonged therapy that involves potentially toxic drugs. Several authors firmly believe that this vital distinction, based on clinical and imaging features, is very difficult to make. ${ }^{44}$ In addition to those features suggested by Rajshekhar and Chandy, ${ }^{38}$ several other imaging features have been suggested to differentiate between these two entities. For example, in this setup a target lesion (a lesion with central or eccentric nidus of calcification or a dot of enhancement) is frequently visualized. In the past, these target lesions were considered a pathognomonic feature of CNS tuberculoma. ${ }^{2}$ More recently, Del Brutto, et al. ${ }^{10}$ reported that visualization of an enhancing eccentric dot, which may possibly represent the scolex of cysticercosis, can be considered a definite imaging feature of cysticercus origin.

Magnetic Resonance Imaging. Magnetic resonance imaging is frequently performed with the objective of differentiating between cysticercal granuloma and tuberculoma. In fact, the MR imaging features of both these conditions are also very similar and usually not helpful in this differentiation. Investigation entailing $\mathrm{T}_{1}$-weighted MR images reveals a low signal center with isointense periphery. Granulomas are better visualized on $\mathrm{T}_{2}$-weighted sequences where a low signal ring and high signal center are characteristic features. Surrounding edema is also best seen on $\mathrm{T}_{2}$-weighted MR images (Fig. 4). On contrast-enhanced MR imaging studies the granuloma shows marked peripheral enhancement and a low signal area in the center. ${ }^{36}$

Serological Studies. The two principal serological tests are the enzyme-linked immunosorbent assay and the enzyme-linked immunotransfer blot. The latter is regarded as more reliable, with a specificity of $100 \%$ and a sensi- 


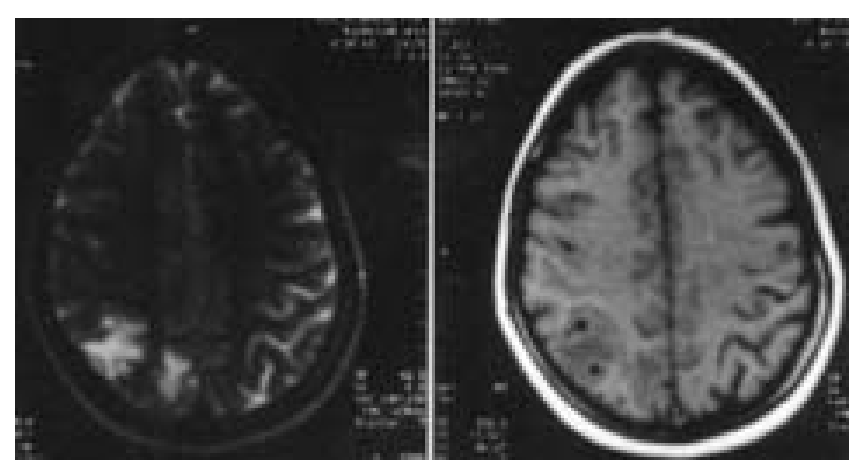

Fig. 4. Magnetic resonance imaging revealing a single enhancing lesion on a $\mathrm{T}_{2}$-weighted sequence (left) and $\mathrm{T}_{1}$-weighted sequence (right).

tivity of up to $97 \%$, in tests of both blood and cerebrospinal fluid. Several authors, however, have demonstrated that these tests display poor sensitivity in detecting antibodies in cases of single lesions when sensitivity is very low (range 14-45\%). Positive results obtained with these tests may help in confirming the diagnosis of cysticercal granuloma, but negative results cannot be used to exclude a diagnosis of cysticercosis. ${ }^{40,41}$

Diagnostic Criteria. Several diagnostic criteria for NCC have been proposed; however, in none of the criteria has the issue of differentiation between cysticercal granuloma and tuberculoma been addressed. This is the most difficult differentiation clinicians face in the developing countries. These diagnostic criteria are based on clinical, epidemiological, imaging, and serological features along with response to anticysticercal drugs. Unfortunately, none of the criteria proposed by Del Brutto, et al., ${ }^{9,10}$ seems to be helpful in differentiating between these two entities (Table 3).

\section{Other Diagnostic Possibilities}

Several other diseases may also pose a diagnostic challenge in patients with a single enhancing CT-documented lesion. It is important to assess for the presence of a systemic disease such as pulmonary tuberculosis, malignancy, or a source of bacterial infection. A small CT-demonstrated enhancing brain lesion in an aged individual should arouse great suspicion of secondary malignancy and effort should be made to define primary site. When applicable (for example, in cases in which there is a history of sexual contact with a high-risk individual), serological tests for the human immunodeficiency virus infection should be performed because several human immunodeficiency virus-related CNS complications such as toxoplasmosis, CNS lymphoma, and various fungal granulomas can present with single enhancing lesions.

Enlargement of lesions on follow-up CT scans is not necessarily indicative of a neoplastic nature because a small but significant number of single cysticercal granuloma may show paradoxical enlargement (exceeding $20 \mathrm{~mm}$ in diameter) after treatment with anticysticercal drugs. ${ }^{37}$ Singh and associates ${ }^{45}$ reported two patients in whom even enlarged CT-evidenced lesions resolved spontaneously.
TABLE 3

Revised diagnostic criteria for $N C C^{*}$

Criteria $\uparrow$

\begin{tabular}{c}
\hline \hline \multicolumn{1}{c}{ Criteria $\uparrow$} \\
\hline absolute \\
histological demonstration of parasite \\
CT or MR evidence of cystic lesion w/ scolex \\
fundoscopic visualization of parasite \\
major \\
lesions suggestive of NCC on CT or MR \\
positive serum EITB $\$$ \\
resolution of cyst after therapy \\
spontaneous resolution of single enhancing lesion \\
minor \\
lesions compatible w/ NCC on CT or MR \\
suggestive clinical feature \\
positive CSF ELISA $\$$ \\
cysticercosis outside CNS \\
epidemiological \\
household contact with T. solium infection \\
emigration from or living in endemic area \\
travel to endemic area
\end{tabular}

* As established by Del Brutto, et al. (2001)

$\dagger$ Definite diagnosis of NCC: one absolute criterion; or two major and one minor and one epidemiological criteria. Probable diagnosis of NCC: one major and two minor criteria; one major and one minor and one epidemiological criteria; or three minor and one epidemiological criteria.

† EITB = enzyme-linked immunotransfer blot; ELISA = enzyme-linked immunosorbent assay.

\section{MANAGEMENT OF THE SINGLE ENHANCING LESION}

Until recently, there was no consensus concerning the exact origin of these single enhancing lesions in India; thus, a uniform treatment has not been practiced. Various physicians continue to treat these lesions differently.

\section{Antituberculous Treatment}

In few initial series, patients received antituberculous treatment..$^{50}$ After histopathological demonstration of cysticercal disease origin in a majority of patients, as well as evidence of spontaneous resolution of these lesions, antituberculous treatment is now infrequently used.

\section{Anticysticercal Treatment}

In Latin American countries, CT-depicted single enhancing lesions are invariably treated either with albendazole or praziquantel. In an uncontrolled study Del Brutto ${ }^{8}$ observed early resolution of lesions on CT scans following treatment with albendazole. More recently, in a controlled study, Pretell, et al., ${ }^{28}$ included 26 patients with single enhancing lesions. The patients were openly assigned to receive either single-day praziquantel therapy (three doses of $25 \mathrm{mg} / \mathrm{kg}$ at 2-hour intervals) or no treatment. In praziquantel-treated patients, complete resolution occurred in 11 and partial resolution in two; in the remaining patient the lesion was later diagnosed as AVM. Conversely, the lesions persisted unchanged in six of 12 patients in the nontreatment group. The authors favored routine administration of anticysticercal drugs in patients with single enhancing lesions. ${ }^{28}$ Although this single-day praziquantel therapy has been found particularly useful for single le- 
sions, poor response has been noted in those with multiple cysticercal lesions. ${ }^{29}$

In India, studies involving anticysticercal treatment have provided conflicting results. In a placebo-controlled study Padma, et al., ${ }^{27}$ observed that 7-day treatment with albendazole did not hasten the resolution of CT-documented lesions. In a different double-blind placebocontrolled study, however, Baranwal, et al., ${ }^{1}$ observed a significantly faster and higher incidence of complete disappearance of lesions in children who underwent 28-day albendazole treatment $(15 \mathrm{mg} / \mathrm{kg} /$ day $)$. The conflicting results of these two studies also fueled the controversy of the ideal dosage regimen of albendazole. A comparative study is needed to evaluate 7- or 8-day albendazole treatment in a 30-day course in patients with single CT-enhancing lesion. In patients with other forms of NCC, Cruz, et al., ${ }^{7}$ have already demonstrated that 8 day albendazole treatment is as effective as 15- or 30-day therapy. These authors concluded that there is no benefit to extending albendazole treatment beyond 7 or 8 days.

\section{Antiepileptic Treatment}

It has been argued that because CT-demonstrated enhancing lesions represent dying stages of cysticercal lesion, they require no anticysticercal treatment. Because these lesions disappear spontaneously, patients require only antiepileptic drugs to control the associated seizure disorder. ${ }^{12}$ Chopra, et al., ${ }^{6}$ reported on 78 patients who underwent follow-up repeated CT scanning within 6 to 12 weeks; in 47 cases complete spontaneous resolution of the lesions was observed. Significant reduction in the size of the lesions and surrounding cerebral edema occurred in 24 patients; additional repeated CT studies revealed either complete disappearance or considerable regression in due course. All these patients received antiepileptic drugs only. In the only prospective study, Singh, et al., ${ }^{46}$ included 75 patients with single enhancing lesions. Follow-up CT scanning conducted after 2 months revealed complete spontaneous disappearance of lesions in 55 patients (73.3\%). In 11 patients $(14.7 \%)$ the lesions became calcified. In the remaining nine patients in whom the lesions persisted or regressed, another follow-up CT study (after 6 months) revealed either complete disappearance or calcification. The majority of patients $(87 \%)$ remained seizure free after 1-year follow-up examination.

\section{Associated Seizure Disorder}

Enough evidence is available to suggest that the prognosis of associated seizure disorder is better in cases in which single enhancing lesions are present than in those in which other forms of parenchymal NCC are found; in the latter there is very high incidence of seizure recurrence after withdrawal of antiepileptic drugs. ${ }^{3}$ It has been suggested that patients with CT-documented single enhancing lesions do not require conventional prolonged therapy of 2 to 3 years. Antiepileptic drugs can safely be withdrawn once the lesion has disappeared. In a retrospective study, Murthy and Subba Reddy ${ }^{25}$ studied 102 patients in whom CT scanning revealed a single enhancing lesion and in whom seizures were present. In 64 patients seizures did not recur once antiepileptic treatment was instigated.
Twenty-eight patients $(27.5 \%)$ continued to experience seizure recurrence for a median 2-month period before spontaneous remission was achieved. In the remaining 10 patients seizures recurred after albendazole therapy (median period of seizure recurrence 8 months). The antiepileptic drugs were withdrawn in all patients after the follow-up CT scan revealed complete resolution of the lesions. After withdrawal of antiepileptic drugs, only one patient experienced seizure recurrence during the mean follow-up period of 45 months (range 19-101 months). In this patient in whom seizures recurred, follow-up CT scanning revealed a gliotic scar at the site of the enhancing lesion. ${ }^{25}$

In one review Carpio, et al., ${ }^{3}$ suggested that seizures in patients with CT-demonstrated single enhancing lesions should be considered as acute symptomatic seizure disorder and such patients require antiepileptic treatment for the acute-stage period (perhaps for several months, during which the inflammatory reaction of the lesion is most active). Once the lesion has disappeared, antiepileptic therapy may be gradually withdrawn. If seizures recur, the antiepileptic therapy treatment should be reinitiated and the patient may be treated for 2 to 3 years. If lesions have become calcified, the antiepileptic therapy should be provided for a longer period (2-3 years) before being tapered.

\section{PERSISTENCE OF THE ENHANCING LESIONS}

Until recently, if single enhancing lesions did not disappear or regress within a reasonable time period (usually within 6 months), they were viewed with suspicion (Fig. 5). Often alternative diagnoses such as tuberculoma, pyogenic abscesses, or metastatic lesions were considered. Some authors have contended that persistence of lesions indicates that more aggressive treatment brain biopsy sampling is necessary. ${ }^{31}$ Currently it is very difficult to set a cutoff period after which these lesions may be termed "persisting." In a recent prospective follow-up study, Rajshekhar ${ }^{32}$ noted that the longer the follow-up period the higher the number of cases in which spontaneous disappearance of the granuloma occurred. He observed that at 6 months in only $19 \%$ of 210 patients had complete resolution occurred whereas at the end of 1 year and 2 years,

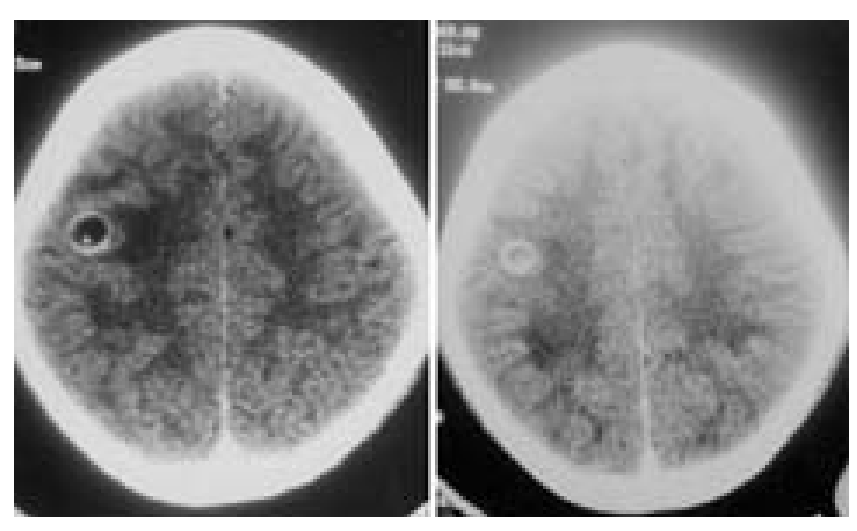

Fig. 5. Left: Contrast-enhanced CT scan demonstrating a ringenhancing lesion. Right: Two-year follow-up CT scan demonstrating the persisting lesion. 
respectively, in approximately 63 and $89 \%$ of patients CT scans revealed normal findings. Garg and Nag ${ }^{16}$ also reported similar observations in a retrospective study. They observed that in 16 of 101 patients the lesions did not disappear or regress after 6 months. Additional follow-up scans in these 16 patients, however, revealed that the lesion eventually calcified in four patients, the ring lesions changed to disc lesion and degree of associated edema was considerably less in four, and the lesion persisted unchanged in the remaining eight. ${ }^{16}$ Although concern is often expressed, in none of the prospective and retrospective follow-up studies has either clinical deterioration or significant enlargement of lesions been noted. ${ }^{6,15,25,26,32,46}$ In several uncontrolled series, albendazole therapy has been shown to produce complete resolution of persisting lesions. ${ }^{30,49}$

\section{ROLE OF NEUROSURGERY}

The mainstay of treatment in patients with CT-evidenced single enhancing lesions is seizure control with antiepileptic drugs. Seizures can often be very well controlled. Small cortical granulomas do not require biopsy sampling or removal of the lesion because the parasite is in the stage of dying and will disappear spontaneously. The principal indications for surgical intervention in patients with NCC are treatment of hydrocephalus, the removal of mobile intraventricular cysts, spinal cysts, acces- sible racemose cysts in the basal cisterns, and large supratentorial cysts causing mass effects.

Stereotactic brain biopsy sampling is often difficult because of the toughness and mobility of the cysticercal lesion, and it can also be hazardous because of the typical site of lesions at the junction of gray-white matter and possible risk of intracerebral hemorrhage. Moreover, because the lesion is benign, obtaining a biopsy sample is not justified in every patient. In very rare cases in which a lesion enlarges and causes increasing neurological deficit refractory to albendazole treatment, other diagnoses such as abscess, tuberculoma, or tumor (primary or secondary metastasis) are likely. Later in the course neurosurgery may be required. Even in developed countries where these lesions are infrequent this same nonsurgical approach has proven successful. ${ }^{51}$

\section{CONCLUSIONS}

Initially single enhancing lesions demonstrated on CT scanning were presumed to be tuberculoma and were treated with empirical antituberculous drugs. Later, histopathological examination of brain tissue biopsy samples suggested that, in majority, these lesions are single cysticercal granuloma. Tuberculoma may be present in a few of these patients. It is very difficult to differentiate cysticercal granuloma from tuberculoma based on clinical and radiological characteristics. The authors of several pro-

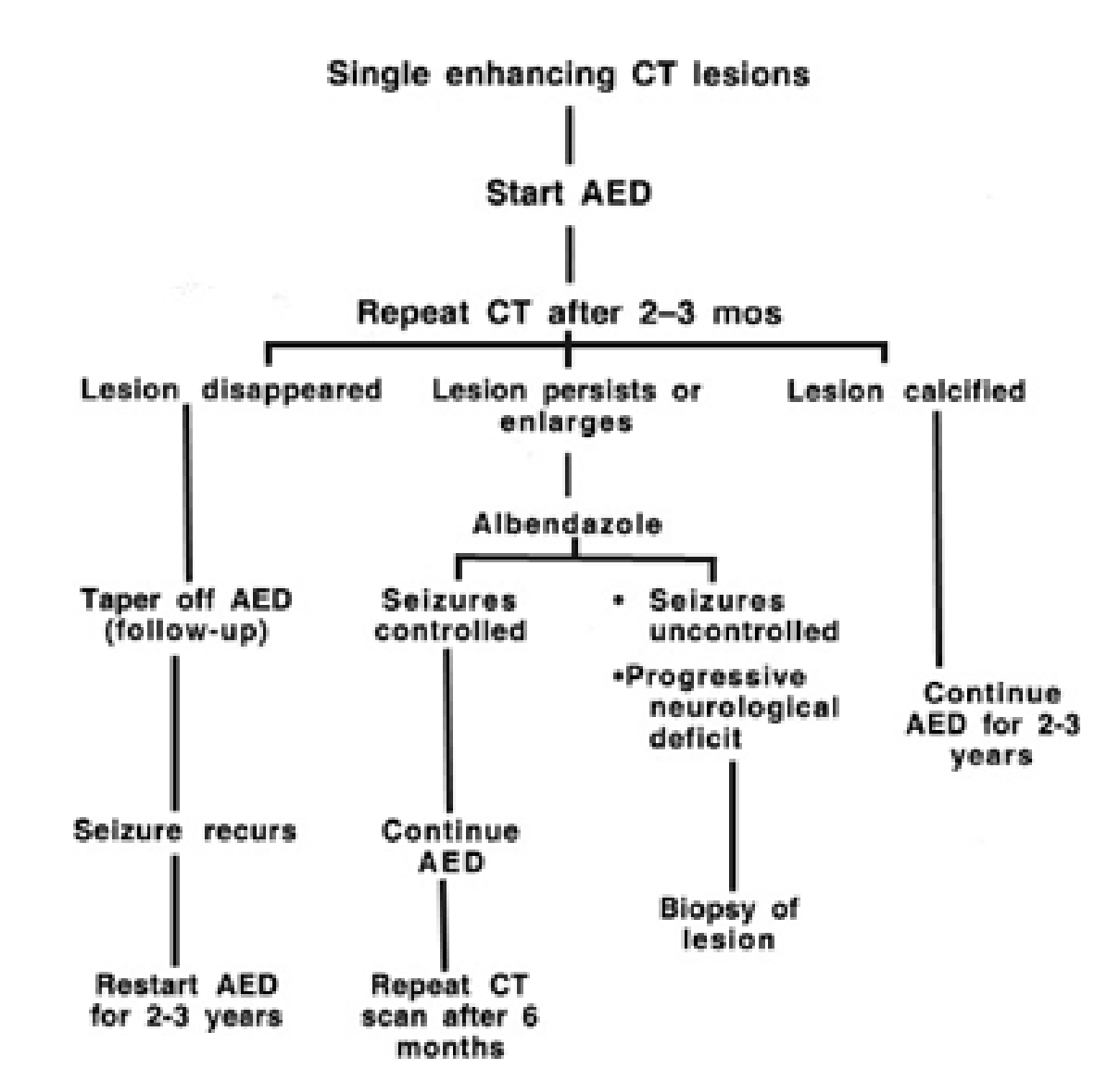

Fig. 6. Treatment algorithm for the management of patients with CT-documented single enhancing lesions. AED = antiepileptic drug. 
spective and retrospective studies have convincingly demonstrated that, regardless of origin, single enhancing CTdocumented lesions tend to disappear spontaneously. $\mathrm{Pa}-$ tients require antiepileptic drugs to prevent seizure recurrence. The role of albendazole or praziquantel in hastening the resolution of lesions is uncertain. Antiepileptic drugs may safely be withdrawn after CT evidence that the lesion has disappeared. Neurosurgical intervention may be considered if lesions increase in size and produce uncontrolled seizure or progressive focal neurological deficit (Fig. 6).

\section{References}

1. Baranwal AK, Singhi PD, Khandelwal N, et al: Albendazole therapy in children with focal seizures and single small enhancing computerized tomographic lesions: a randomized placebocontrolled double blind trial. Pediatr Infect Dis J 17:696-700, 1998

2. Bargallo J, Berenguer J, Garcia-Barrionuevo J, et al: The "target sign": is it a specific sign of CNS tuberculoma? Neuroradiology 38:547-550, 1996

3. Carpio A, Escobar A, Hauser WA: Cysticercosis and epilepsy: a critical review. Epilepsia 39:1025-1040, 1998

4. Chacko G, Rajshekhar V, Chandy MJ, et al: The calcified intracorporeal vacuole: an aid to the pathological diagnosis of solitary cerebral cysticercus granulomas. J Neurol Neurosurg Psychiatry 69:525-527, 2000

5. Chandy MJ, Rajshekhar V, Prakash S, et al: Cysticercosis causing single, small CT lesions in Indian patients with seizures. Lancet 1:390-391, 1989

6. Chopra JS, Sawhney IMS, Suresh N, et al: Vanishing CT lesions in epilepsy. J Neurol Sci 107:40-49, 1992

7. Cruz I, Cruz ME, Carrasco F, et al: Neurocysticercosis: optimal dose treatment with albendazole. J Neurol Sci 133:152-154, 1995

8. Del Brutto $\mathrm{OH}$ : The use of albendazole in patients with single lesions enhanced on contrast CT. N Engl J Med 328:356-357, 1993

9. Del Brutto OH, Rajshekhar V, White AC Jr, et al: Proposed diagnosed criteria of neurocysticercosis. Neurology 157: 177-183, 2001

10. Del Brutto OH, Wadia NH, Dumas M, et al: Proposal of diagnostic criteria for human cysticercosis and neurocysticercosis. $\mathbf{J}$ Neurol Sci 142:1-6, 1996

11. Escobar A: The pathology of neurocysticercosis, in Palacios E, Rodriguez-Carbajal J, Taveras JM (eds): Cysticercosis of the Central Nervous System. Springfield, IL: Charles C Thomas, 1983, pp 27-54

12. Garg RK: Solitary cysticercus granulomas. J Neurosurg 82: 911-912, 1995 (Letter)

13. Garg RK, Karak B, Sharma AM, et al: Single CT (ring) lesion in epilepsy patients: a new observation. Indian J Pediatr 66: 155-157, 1999

14. Garg RK, Misra S: Non-epileptic manifestations in patients with single enhancing computed tomography lesions. J Assoc Physicians India 48:788-793, 2000

15. Garg RK, Nag D: Single enhancing CT lesions in Indian patients with seizures: clinical and radiological evaluation and followup. J Trop Pediatr 44:204-210, 1998

16. Garg RK, Nag D: Single ring- or disk-enhancing computed tomographic lesion in Indian children and adolescents after first seizure. Arch Pediatr Adolesc Med 157:632-634, 1997

17. Garg RK, Singh MK, Misra S: Single-enhancing CT lesions in Indian patients with seizures: a review. Epilepsy Res 38: 91-104, 2000

18. Goulatia RK, Verma A, Misra NK, et al: Disappearing CT lesions in epilepsy. Epilepsia 28:523-527, 1987
19. Jayakumar PN, Taly AB, Mohan PK: Transient computerised tomographic (CT) abnormalities following partial seizures. Acta Neurol Scand 72:26-29, 1985

20. Kennedy A, Schon F: Epilepsy: disappearing lesions appearing in the United Kingdom. BMJ 302:933-935, 1991

21. Kumar R, Kumar A, Kohli N, et al: Ring or disc-like enhancing lesions in partial epilepsy in India. J Trop Pediatr 36:131-134, 1990

22. Lath R, Rajshekhar V: Solitary cysticercus granuloma of the brainstem. Report of four cases. J Neurosurg 89:1047-1051, 1998

23. Minguetti G, Ferreira MV: Computed tomography in neurocysticercosis. J Neurol Neurosurg Psychiatry 46:936-942, 1983

24. Misra S, Verma R, Lekhra OP, et al: CT observations in partial seizures. Neurol India 42:24-27, 1994

25. Murthy JMK, Subba Reddy YV: Prognosis of epilepsy associated with single CT enhancing lesion: a long term follow up study. J Neurol Sci 159:151-155, 1998

26. Murthy JMK, Yangala R, Srinivas M: The syndromic classification of the International League Against Epilepsy: a hospitalbased study from South India. Epilepsia 39:48-54, 1998

27. Padma MV, Behari M, Misra NK, et al: Albendazole in single CT ring lesions in epilepsy. Neurology 44:1344-1346, 1994

28. Pretell EJ, Garcia HH, Custodio N, et al: Short regimen of praziquantel in the treatment of single brain enhancing lesions. Clin Neurol Neurosurg 102:215-218, 2000

29. Pretell EJ, Garcia HH, Gilman RH, et al: Failure of one-day praziquantel treatment in patients with multiple neurocysticercosis lesions. Clin Neurol Neurosurg 103:175-177, 2001

30. Rajshekhar V: Albendazole therapy of persistent, solitary cysticercus granulomas in patients with seizures. Neurology 43: 1238-1240, 1993

31. Rajshekhar V: Etiology and management of single small CT lesions in patients with seizures: understanding a controversy. Acta Neurol Scand 84:465-470, 1991

32. Rajshekhar V: Rate of spontaneous resolution of a solitary cysticercus granuloma in patients with seizures. Neurology 57: 2315-2317, 2001

33. Rajshekhar V: Severe episodic headache as the sole presenting ictal event in patients with a solitary cysticercus granuloma. Acta Neurol Scand 102:44-46, 2000

34. Rajshekhar V, Chacko G, Haran RP, et al: Clinicoradiological and pathological correlations in patients with solitary cysticercus granuloma and epilepsy: focus on presence of the parasite and oedema formation. J Neurol Neurosurg Psychiatry 59: 284-286, 1995

35. Rajshekhar V, Chandy MJ: Clinical manifestation of solitary cysticercus granuloma, in Chandy MJ, Rajshekhar V (eds): Cysticercus Granuloma: The Disappearing Lesion. Hyderabad: Orient Longman, 2000, pp 29-39

36. Rajshekhar V, Chandy MJ: Comparative study of CT and MRI in patients with seizures and a solitary cerebral cysticercus granuloma. Neuroradiology 38:542-546, 1996

37. Rajshekhar V, Chandy MJ: Enlarging cysticercus granulomas. J Neurosurg 80:840-843, 1994

38. Rajshekhar V, Chandy MJ: Validation of diagnostic criteria for solitary cysticercus granuloma in patients presenting with seizures. Acta Neurol Scand 96:76-81, 1997

39. Rajshekhar V, Haran RP, Prakash GS, et al: Differentiating solitary small cysticercus granulomas and tuberculomas in patients with epilepsy. Clinical and computerized tomographic criteria. J Nerurosurg 78:402-407, 1993

40. Rajshekhar V, Oommen A: Serological studies using ELISA and EITB in patients with solitary cysticercus granuloma and seizures. Neurol Infect Epidemiol 2:177-180, 1997

41. Rajshekhar V, Wilson M, Schantz PM: Cysticercus immunoblot assay in Indian patients with single enhancing small CT lesions. J Neurol Neurosurg Psychiatry 54:561-562, 1991

42. Sethi PK, Kumar BR, Madan VS, et al: Appearing and disap- 


\section{Enhancing radiological lesion}

pearing CT scan abnormalities and seizures. J Neurol Neurosurg Psychiatry 48:866-869, 1985

43. Sethi PP, Wadia RS, Kiyawat DP, et al: Ring or disc enhancing lesions in epilepsy in India. J Trop Med Hyg 97:347-353, 1994

44. Shah GV: Central nervous system tuberculosis: imaging manifestations. Neuroimaging Clin N Am 10:355-374, 2000

45. Singh G, Sobti P, Bhatia RS, et al: Enlarging single CT lesions can also spontaneously resolve. Neurol India 48:85-87, 2000

46. Singh MK, Garg RK, Nath G, et al: Single small enhancing computed tomographic (CT) lesions in Indian patients with newonset seizures. A prospective follow-up in 75 patients. Seizure 10:573-578, 2001

47. Stamos JK, Rowley AH, Hahn YS, et al: Neurocysticercosis: report of unusual pediatric cases. Pediatrics 98:974-977, 1996

48. Tandon PN, Bhargava S: CNS tuberculosis: lessons learnt from CT studies. Neurol India 28:207-212, 1980

49. Thussu A, Arora A, Lal V, et al: Albendazole therapy for soli- tary persistent cysticercus granuloma. Neurol India 49:95-97, 2001

50. Wadia RS, Makhale CN, Kelkar AV, et al: Focal epilepsy in India with special reference to lesions showing ring or disc-like enhancement on contrast computed tomography. J Neurol Neurosurg Psychiatry 50:1298-1301, 1987

51. Wadley JP, Shakir RA, Rice Edwards JM: Experience with neurocysticercosis in the UK: correct diagnosis and neurosurgical management of the small enhancing brain lesion. Br J Neurosurg 14:211-218, 2000

Manuscript received April 15, 2002.

Accepted in final form June 4, 2002.

Address reprint requests to: Ravindra Kumar Garg, M.D., D.M., Department of Neurology, King George's Medical College, Lucknow-226003, India. email: garg50@yahoo.com. 